\title{
CONDITIONAL CERTAINTY EQUIVALENT
}

\author{
MARCO FRITTELLI* and MARCO MAGGIS ${ }^{\dagger}$ \\ Department of Mathematics, University of Milan \\ via C. Saldini 50 Milan, 20134, Italy \\ *marco.frittelli@unimi.it \\ ${ }^{\dagger}$ marco.maggis@unimi.it
}

Received 26 March 2010

Accepted 17 May 2010

\begin{abstract}
In a dynamic framework, we study the conditional version of the classical notion of certainty equivalent when the preferences are described by a stochastic dynamic utility $u(x, t, \omega)$. We introduce an appropriate mathematical setting, namely Orlicz spaces determined by the underlying preferences and thus provide a systematic method to go beyond the case of bounded random variables. Finally we prove a conditional version of the dual representation which is a crucial prerequisite for discussing the dynamics of certainty equivalents.
\end{abstract}

Keywords: Stochastic dynamic utility; conditional certainty equivalent; Musielak-Orlicz spaces; quasiconcavity; dual representation.

\section{Introduction}

In the last decade many methodologies for pricing in incomplete markets were build on expected utility maximization with respect to terminal wealth: classic examples of this approach are the notions of fair price [8], certainty equivalent [14] and indifference price $[4,9,16]$.

These techniques were developed both in a static framework and in a dynamic context [10]. In the dynamic case however, the utility function represents preferences at a fixed time $\mathrm{T}$, while the pricing occurs at any time between today and the expiration $\mathrm{T}$ (backward pricing). The martingale property of the indirect utility (the value function of the optimization problem [11]) is an automatic consequence of the dynamic programming principle.

This classic backward approach has recently been argued in $[3,15,22,23]$ and a novel forward theory has been proposed: the utility function is stochastic, time dependent and moves forward.

In this theory, the forward utility (which replaces the indirect utility of the classic case) is built through the underlying financial market and must satisfy some appropriate martingale conditions.

${ }^{\dagger}$ Corresponding author. 
Our research is inspired by the theory just mentioned, but a different approach is here developed: our preliminary object will be a stochastic dynamic utility $u(x, t, \omega)$ - i.e. a stochastic field [19] — representing the evolution of the preferences of the agent (see Definition 1.1).

The definition of the Conditional Certainty Equivalent (CCE) that we propose and analyze in this paper (Definition 1.6), is the natural generalization to the dynamic and stochastic environment of the classical notion of the certainty equivalent, as given in [25]. The CCE, denoted by $C_{s, t}(\cdot)$, provides the time $s$ value of an $F_{t}$ measurable claim $(s \leq t)$ in terms only of the Stochastic Dynamic Utility (SDU) and the filtration.

The SDU that we consider does not require a priori the presence of a financial market; neither it will have any specific recursive structure, nor will necessarily be an indirect utility function based on optimal trading in the market. However, as studied in Sec. 1.1, appropriate conditions are required on the SDU in order to deduce interesting properties for the CCE.

The next step, which is left for future research, would be the investigation of the compatibility conditions between the value assigned by the CCE and existing prices when an underlying market indeed exists. Clearly, not all SDU are compatible with the market. One extreme case is when the SDU can be determined by the market and the initial preferences structure, as in the case of the forward utility theory.

The second part of the paper is devoted to the dual representation of the CCE, considered as a monotone and quasiconcave operator between vector lattices (see Sec. 3). Recently, the dual representation of such operators has been obtained in [13]. Due to the particular form of the CCE, we are able to provide here a direct proof which is also less demanding than the general one.

However, in order to show the dual representation of the CCE we must first define it on appropriate vector lattices. A common approach is to restrict the view to bounded random variables, so that no further integrability conditions are requested. But as soon as we try to extend the scenario to unbounded random variables it immediately appears that the distortion provoked by utility function can be mastered only in ad hoc frameworks.

To this end we introduce in Sec. 2, in the spirit of [5], a generalized class of Orlicz spaces which are naturally associated to the SDU taken into account. We show (Sec. 2.3) with some examples that these spaces also play a fundamental role for time compatibility of the CCE, since $C_{s, t}: M^{\widehat{u}_{t}} \rightarrow M^{\widehat{u}_{s}}$, where $M^{\widehat{u}_{t}}$ is the generalized Orlicz space of $\mathcal{F}_{t}$ measurable random variables associated to the SDU $u(x, t, \omega)$.

\subsection{Definitions and first properties}

A non-atomic probability space $(\Omega, \mathcal{F}, \mathbb{P})$ and a right continuous filtration $\left\{\mathcal{F}_{t}\right\}_{t \geq 0}$ are fixed throughout this paper. As usual we denote with $L^{0}(\Omega, \mathcal{F}, \mathbb{P})$ the space of $\mathcal{F}$ measurable random variables that are $\mathbb{P}$ almost surely finite and with $\bar{L}^{0}(\Omega, \mathcal{F}, \mathbb{P})$ 
the space of extended r.v. which take values in $\mathbb{R} \cup\{\infty\}$. The $L^{p}(\Omega, \mathcal{F}, \mathbb{P})$ spaces, $p \in[0, \infty]$, will simply be denoted by $L^{p}$, unless it is necessary to specify the sigma algebra, in which case we write $L_{\mathcal{F}}^{p}$. All equalities and inequalities are intended to be $\mathbb{P}$ almost surely: by $X>Y$ we mean that $\mathbb{P}(X>Y)=1$. Otherwise, for any $\mathbb{Q} \ll \mathbb{P}$ we explicitly write $X>_{\mathbb{Q}} Y$. Moreover the essential ( $\mathbb{P}$ a.s.) supremum ess $\sup \left(X_{\lambda}\right)$ of a family of random variables $X_{\lambda}$ will be simply denoted by $\sup \left(X_{\lambda}\right)$, and similarly for the essential infimum (see [12] Sec. A.5 for reference). Hereafter the symbol $\hookrightarrow$ denotes inclusion and lattice embedding between two lattices, and $\vee($ resp. $\wedge$ ) the essential $(\mathbb{P}$ a.s.) maximum (resp. the essential minimum) between two random variables, which are the usual lattice operations.

Definition 1.1. A stochastic dynamic utility (SDU)

$$
u: \mathbb{R} \times[0, \infty) \times \Omega \rightarrow \mathbb{R} \cup\{-\infty\}
$$

satisfies the following conditions: for any $t \in[0,+\infty)$ there exists $A_{t} \in \mathcal{F}_{t}$ such that $\mathbb{P}\left(A_{t}\right)=1$ and

(a) the effective domain, $\mathcal{D}(t):=\{x \in \mathbb{R}: u(x, t, \omega)>-\infty\}$ and the range $\mathcal{R}(t):=$ $\{u(x, t, \omega) \mid x \in \mathcal{D}(t)\}$ do not depend on $\omega \in A_{t}$; moreover $0 \in \operatorname{int} \mathcal{D}(t)$, $E[u(0, t)]<+\infty$ and $\mathcal{R}(t) \subseteq \mathcal{R}(s) ;$

(b) for all $\omega \in A_{t}$ and $t \in[0,+\infty)$ the function $x \rightarrow u(x, t, \omega)$ is strictly increasing on $\mathcal{D}(t)$ and increasing, concave and upper semicontinuous on $\mathbb{R}$.

(c) $\omega \rightarrow u(x, t, \cdot)$ is $\mathcal{F}_{t}$-measurable for all $(x, t) \in \mathcal{D}(t) \times[0,+\infty)$

The following assumption may turn out to be relevant in the sequel of the paper, even if not necessary for the definition of SDU.

(d) For any fixed $x \in \mathcal{D}(t), u(x, t, \cdot) \leq u(x, s, \cdot)$ for every $s \leq t$.

Remark 1.1. We identify two SDU, $u \sim \widetilde{u}$, if for every $t \in[0,+\infty)$, the two domains are equal $(\mathcal{D}(t)=\widetilde{\mathcal{D}}(t))$ and there exists an $\mathcal{F}_{t}$-measurable set $B_{t}$ such that $\mathbb{P}\left(B_{t}\right)=1$ and $u(x, t, \omega)=\widetilde{u}(x, t, \omega)$ for every $(x, \omega) \in \mathcal{D}(t) \times B_{t}$.

In the sequel, we denote $u(x, t, \cdot)$ simply by $u(x, t)$, unless confusion may arise.

In order to define the conditional certainty equivalent we introduce the set

$$
\mathcal{U}(t)=\left\{X \in L^{0}\left(\Omega, \mathcal{F}_{t}, \mathbb{P}\right) \mid u(X, t) \in L^{1}(\Omega, \mathcal{F}, \mathbb{P})\right\} .
$$

Lemma 1.1. Let $u$ be a $S D U$.

(i) (Inverse) Let $t \in[0, \infty)$ and $A_{t} \in \mathcal{F}_{t}$ as in Definition 1.1: the inverse function $u^{-1}: \mathcal{R}(t) \times[0, \infty) \times A_{t} \rightarrow \mathcal{D}(t)$

$$
u^{-1}(u(x, t, \omega), t, \omega)=x
$$

is well defined. For each $\omega \in A_{t}$, the function $u^{-1}(\cdot, t, \omega)$ is continuous and strictly increasing on $\mathcal{R}(t)$ and $u^{-1}(y, t, \cdot)$ is $\mathcal{F}_{t}$-measurable for all $y \in \mathcal{R}(t)$. 
(ii) (Comparison) Fix any $t \in[0, \infty)$; if $X, Y \in \mathcal{U}(t)$ then $u(X, t) \leq u(Y, t)$ if and only if $X \leq Y$. The same holds if the inequalities are replaced by equalities.

(iii) (Jensen) If $X \in L_{\mathcal{F}_{t}}^{1}$ and $u(X, s)$ is integrable, then, for all $s \leq t$,

$$
E\left[u(X, s) \mid \mathcal{F}_{s}\right] \leq u\left(E\left[X \mid \mathcal{F}_{s}\right], s\right)
$$

(iv) (Extended Jensen) Suppose $u(x, s)$ is integrable for every $x \in \mathcal{D}(s)$. Let $X \in$ $L_{\mathcal{F}_{t}}^{0}$, such that $u(X, s)^{-}$is integrable. Then

$$
E\left[u(X, s) \mid \mathcal{F}_{s}\right] \leq u\left(E\left[X \mid \mathcal{F}_{s}\right], s\right)
$$

where the conditional expectation is meant in an extended way.

Proof. (i) Since both assumptions (a) and (b) hold on $A_{t}$, the existence of a continuous, increasing inverse function follows straightforwardly. From assumption (c) we can deduce that $u^{-1}(y, t, \cdot)$ is $\mathcal{F}_{t}$-measurable for all $y \in \mathcal{R}(t)$.

(ii) Is also immediate since $u$ is strictly increasing as a function of $x$.

(iii) This property follows from the Theorem p.79 in [21].

(iv) First we suppose that $u(0, s)=0$. This implies that $u(X, s) \mathbf{1}_{A}=u\left(X \mathbf{1}_{A}, s\right)$ for every $A \in \mathcal{F}_{t}$. Recall that if $Y \in L_{\mathcal{F}_{t}}^{0}$ and $Y \geq 0$ then $E\left[Y \mid \mathcal{F}_{s}\right]:=$ $\lim _{n} E\left[Y \mathbf{1}_{Y \leq n} \mid \mathcal{F}_{s}\right]$ is well defined.

First we show that $u(X, s)^{-}$integrable implies $E\left[X \mathbf{1}_{\{X<0\}} \mid \mathcal{F}_{s}\right]>-\infty$ and therefore both terms in (1.2) are well defined. From the equality $-u(X, s) \mathbf{1}_{\{X<0\}}=$ $u(X, s)^{-}$we get that $u(X, s) \mathbf{1}_{\{X<0\}}$ is integrable. From (iii) we have that $u(0, s) \geq$ $u\left(X 1_{\{0>X \geq-n\}}, s\right) \geq u(-n, s)$ implies:

$$
E\left[u\left(X \mathbf{1}_{\{0>X \geq-n\}}, s\right) \mid \mathcal{F}_{s}\right] \leq u\left(E\left[X \mathbf{1}_{\{0>X \geq-n\}} \mid \mathcal{F}_{s}\right], s\right) .
$$

By monotone convergence, from (1.3) we then get our claim:

$$
-\infty<E\left[u\left(X \mathbf{1}_{\{X<0\}}, s\right) \mid \mathcal{F}_{s}\right] \leq u\left(E\left[X \mathbf{1}_{\{X<0\}} \mid \mathcal{F}_{s}\right], s\right)
$$

Applying (iii) in the second inequality below we get:

$$
\begin{aligned}
E\left[u(X, s) \mid \mathcal{F}_{s}\right] & =\lim _{n} E\left[u(X, s) \mathbf{1}_{\{0 \leq u(X, s) \leq n\}} \mid \mathcal{F}_{s}\right]+E\left[u(X, s) \mathbf{1}_{\{u(X, s)<0\}} \mid \mathcal{F}_{s}\right] \\
& \leq \lim _{n} E\left[u(X, s) \mathbf{1}_{\{0 \leq X \leq n\}} \mid \mathcal{F}_{s}\right]=\lim _{n} E\left[u\left(X \mathbf{1}_{\{0 \leq X \leq n\}}, s\right) \mid \mathcal{F}_{s}\right] \\
& \leq \lim _{n} u\left(E\left[X \mathbf{1}_{\{0 \leq X \leq n\}} \mid \mathcal{F}_{s}\right], s\right)=u\left(E\left[X^{+} \mid \mathcal{F}_{s}\right], s\right) .
\end{aligned}
$$

Notice that on the $\mathcal{F}_{s}$-measurable set $G^{\infty}:=\left\{E\left[X \mid \mathcal{F}_{s}\right]=+\infty\right\}$ the equation (1.2) is trivial. Since $E\left[-X^{-} \mid \mathcal{F}_{s}\right]>-\infty$, it is clear that $E\left[|X| \mid \mathcal{F}_{s}\right]=+\infty$ on a set $A \in \mathcal{F}$ iff $E\left[X \mid \mathcal{F}_{s}\right]=+\infty$ on the same set $A$. Therefore, by defining $G_{n}:=\{\omega \in$ $\left.\Omega \backslash G^{\infty} \mid E\left[|X| \mid \mathcal{F}_{s}\right](\omega) \leq n\right\}$, we have: $G_{n} \uparrow \Omega \backslash G^{\infty}$. Since each $G_{n}$ is $\mathcal{F}_{s}$-measurable, 
the inequality (1.4)-(1.5) guarantees that

$$
\begin{aligned}
-E\left[u\left(X \mathbf{1}_{G_{n}}, s\right)^{-} \mid \mathcal{F}_{s}\right] & \leq E\left[u\left(X \mathbf{1}_{G_{n}}, s\right) \mid \mathcal{F}_{s}\right] \leq u\left(E\left[X^{+} \mathbf{1}_{G_{n}} \mid \mathcal{F}_{s}\right], s\right) \\
& \leq u\left(E\left[|X| \mid \mathcal{F}_{s}\right], s\right) \mathbf{1}_{G_{n}} \leq u(n, s)
\end{aligned}
$$

and therefore $u\left(X \mathbf{1}_{G_{n}}, s\right)$ is integrable. Obviously, $X \mathbf{1}_{G_{n}}$ is also integrable and we may apply (iii) (replacing $X$ with $X \mathbf{1}_{G_{n}}$ ) and deduce

$E\left[u(X, s) \mid \mathcal{F}_{s}\right] \mathbf{1}_{G_{n}}=E\left[u\left(X \mathbf{1}_{G_{n}}, s\right) \mid \mathcal{F}_{s}\right] \leq u\left(E\left[X \mathbf{1}_{G_{n}} \mid \mathcal{F}_{s}\right], s\right)=u\left(E\left[X \mid \mathcal{F}_{s}\right], s\right) \mathbf{1}_{G_{n}}$.

The thesis follows immediately by taking the limit as $n \rightarrow \infty$, since $G_{n} \uparrow \Omega \backslash G^{\infty}$.

For a general $u(x, s)$, apply the above argument to $v(x, s)=: u(x, s)-u(0, s)$.

A SDU allows us to define the backward conditional certainty equivalent, that represents the time- $s$-value of the time- $t$-claim $X$, for $0 \leq s \leq t<\infty$.

Definition 1.2 (Conditional Certainty Equivalent). Let $u$ be a SDU. The backward Conditional Certainty Equivalent $C_{s, t}(X)$ of the random variable $X \in$ $\mathcal{U}(t)$, is the random variable in $\mathcal{U}(s)$ solution of the equation:

$$
u\left(C_{s, t}(X), s\right)=E\left[u(X, t) \mid \mathcal{F}_{s}\right] .
$$

Thus the CCE defines the valuation operator

$$
\left.C_{s, t}: \mathcal{U}(t) \rightarrow \mathcal{U}(s), \quad C_{s, t}(X)=u^{-1}\left(E\left[u(X, t) \mid \mathcal{F}_{s}\right]\right), s\right) .
$$

Observe that $E\left[u\left(C_{s, t}(X), s\right)\right]=E[u(X, t)]$ and so indeed $C_{s, t}(X) \in \mathcal{U}(s)$.

\section{The definition is well posed:}

(1) For any given $X \in \mathcal{U}(t), E\left[u(X, t) \mid \mathcal{F}_{s}\right] \in L^{1}\left(\Omega, \mathcal{F}_{s}, P\right)$.

(2) Choose two arbitrary versions of the conditional expectation and of the SDU at time $s$, namely $\widetilde{E}\left[u(X, t) \mid \mathcal{F}_{s}\right], \widehat{E}\left[u(X, t) \mid \mathcal{F}_{s}\right]$ and $\widetilde{u}(x, s), \widehat{u}(x, s)$.

(3) For all $\omega \in A_{t}, \underset{\widetilde{E}}{\widetilde{\widetilde{C}}}\left[u(X, t) \mid \mathcal{F}_{s}\right](\omega) \in \mathcal{R}(t) \subseteq \mathcal{R}(s)$. We find a unique solution of $\widetilde{u}\left(\widetilde{C}_{s, t}(X), s\right)=\widetilde{E}\left[u(X, t) \mid \mathcal{F}_{s}\right]$ defined as

$$
\widetilde{C}_{s, t}(X)(\omega)=\widetilde{u}^{-1}\left(\widetilde{E}\left[u(X, t)|| \mathcal{F}_{s}\right](\omega), s, \omega\right) \quad \forall \omega \in A_{t} .
$$

(4) Repeat the previous argument for the second version and find $\widehat{C}_{s, t}(X)$ which differs from $\widetilde{C}_{s, t}(X)$ only on a $\mathbb{P}$-null set.

We could equivalently reformulate the definition of the CCE as follows:

Definition 1.3. The conditional certainty equivalent process is the only process $\left\{Y_{s}\right\}_{0 \leq s \leq t}$ such that $Y_{t} \equiv X$ and the process $\left\{u\left(Y_{s}, s\right)\right\}_{0 \leq s \leq t}$ is a martingale.

In the following proposition we show some elementary properties of the CCE, which have however very convenient interpretations. In (i) we show the semigroup property of the valuation operator; (iii) show the time consistency of the CCE: if the 
time- $v$-values of two time $t$ claims are equal, then the two values should be equal at any previous time; (iv) and (v) are the key properties to obtain a dual representation of the map $C_{s, t}$ as shown in [13]; property (vi) shows that the expectation of the valuation operator is increasing, as a function of the valuation time $s$ and the second issue expresses the risk aversion of the economic agent.

Proposition 1.1. Let $u$ be a $S D U, 0 \leq s \leq v \leq t<\infty$ and $X, Y \in \mathcal{U}(t)$.

(i) $C_{s, t}(X)=C_{s, v}\left(C_{v, t}(X)\right)$.

(ii) $C_{t, t}(X)=X$.

(iii) If $C_{v, t}(X) \leq C_{v, t}(Y)$ then for all $0 \leq s \leq v$ we have: $C_{s, t}(X) \leq C_{s, t}(Y)$. Therefore, $X \leq Y$ implies that for all $0 \leq s \leq t$ we have: $C_{s, t}(X) \leq C_{s, t}(Y)$. The same holds if the inequalities are replaced by equalities.

(iv) Regularity: for every $A \in F_{s}$ we have

$$
C_{s, t}\left(X \mathbf{1}_{A}+Y \mathbf{1}_{A^{C}}\right)=C_{s, t}(X) \mathbf{1}_{A}+C_{s, t}(Y) \mathbf{1}_{A^{C}}
$$

and then $C_{s, t}(X) \mathbf{1}_{A}=C_{s, t}\left(X \mathbf{1}_{A}\right) \mathbf{1}_{A}$.

(v) Quasiconcavity: the upper level set $\left\{X \in \mathcal{U}_{t} \mid C_{s, t}(X) \geq Y\right\}$ is conditionally convex for every $Y \in L_{\mathcal{F}_{s}}^{0}$.

(vi) Suppose $u$ satisfies $(d)$ and for every $t \in[0,+\infty), u(x, t)$ is integrable for every $x \in \mathcal{D}(t)$. Then $C_{s, t}(X) \leq E\left[C_{v, t}(X) \mid \mathcal{F}_{s}\right]$ and $E\left[C_{s, t}(X)\right] \leq E\left[C_{v, t}(X)\right]$. Moreover $C_{s, t}(X) \leq E\left[X \mid \mathcal{F}_{s}\right]$ and therefore $E\left[C_{s, t}(X)\right] \leq E[X]$.

Proof. By definition:

$$
\begin{array}{ll}
u\left(C_{v, t}(X), v\right) \stackrel{(\cdot)}{=} E\left[u(X, t) \mid \mathcal{F}_{v}\right], & X \in \mathcal{U}(t) \\
u\left(C_{s, t}(X), s\right) \stackrel{(+)}{=} E\left[u(X, t) \mid \mathcal{F}_{s}\right], & X \in \mathcal{U}(t) \\
u\left(C_{s, v}(Z), s\right) \stackrel{(\times)}{=} E\left[u(Z, v) \mid \mathcal{F}_{s}\right], & Z \in \mathcal{U}(v)
\end{array}
$$

(i) Let $Z=C_{v, t}(X)$ and compute:

$$
\begin{gathered}
u\left(C_{s, v}\left(C_{v, t}(X)\right), s\right)=u\left(C_{s, v}(Z), s\right) \stackrel{(\times)}{=} E\left[u(Z, v) \mid \mathcal{F}_{s}\right] \\
\stackrel{(\cdot)}{=} E\left[E\left[u(X, t) \mid \mathcal{F}_{v}\right] \mid \mathcal{F}_{s}\right]=E\left[u(X, t) \mid \mathcal{F}_{s}\right] \stackrel{(+)}{=} u\left(C_{s, t}(X), s\right)
\end{gathered}
$$

(ii) Obvious, since $u\left(C_{t, t}(X), t\right) \stackrel{(\cdot)}{=} E\left[u(X, t) \mid \mathcal{F}_{t}\right] \stackrel{(c)}{=} u(X, t)$.

(iii)

$$
\begin{aligned}
u\left(C_{s, t}(X), s\right) & \stackrel{(+)}{=} E\left[u(X, t) \mid \mathcal{F}_{s}\right]=E\left[E\left[u(X, t) \mid \mathcal{F}_{v}\right] \mid \mathcal{F}_{s}\right] \\
& \stackrel{(\cdot)}{=} E\left[u\left(C_{v, t}(X), v\right) \mid \mathcal{F}_{s}\right] \leq E\left[u\left(C_{v, t}(Y), v\right) \mid \mathcal{F}_{s}\right] \\
& \stackrel{(\cdot)}{=} E\left[E\left[u(Y, t) \mid \mathcal{F}_{v}\right] \mid \mathcal{F}_{s}\right] \stackrel{(+)}{=} u\left(C_{s, t}(Y), s\right) .
\end{aligned}
$$

If $X \leq Y$ then $C_{t, t}(X) \leq C_{t, t}(Y)$ and the statement follows from what we just proved. The same for equalities. 
(iv) Consider every $A \in \mathcal{F}_{s}$ and notice that

$$
\begin{aligned}
C_{s, t}\left(X \mathbf{1}_{A}+Y \mathbf{1}_{A^{C}}\right) & =u^{-1}\left(E\left[u(X, t) \mathbf{1}_{A}+u(Y, t) \mathbf{1}_{A^{C}} \mid \mathcal{F}_{s}\right], s\right) \\
& =u^{-1}\left(E\left[u(X, t) \mid \mathcal{F}_{s}\right] \mathbf{1}_{A}, s\right)+u^{-1}\left(E\left[u(Y, t) \mid \mathcal{F}_{s}\right] \mathbf{1}_{A^{C}}, s\right) \\
& =C_{s, t}(X) \mathbf{1}_{A}+C_{s, t}(Y) \mathbf{1}_{A^{C}}
\end{aligned}
$$

(v) Fix an arbitrary $Y \in L_{\mathcal{F}_{s}}^{0}$ and consider the set $\mathcal{Y}=\left\{X \in \mathcal{U}_{t} \mid C_{s, t}(X) \geq Y\right\}$. Take $X_{1}, X_{2} \in \mathcal{Y}$ and $\Lambda \in L_{\mathcal{F}_{s}}^{0}, 0 \leq \Lambda \leq 1$ :

$$
\begin{aligned}
& E\left[u\left(\Lambda X_{1}+(1-\Lambda) X_{2}, t\right) \mid \mathcal{F}_{s}\right] \\
& \quad \geq \Lambda E\left[u\left(X_{1}, t\right) \mid \mathcal{F}_{s}\right]+(1-\Lambda) E\left[u\left(X_{2}, t\right) \mid \mathcal{F}_{s}\right] \geq u(Y, s)
\end{aligned}
$$

hence we get the thesis composing both sides with $u^{-1}(\cdot, s)$.

(vi)

$$
\begin{aligned}
u\left(C_{s, t}(X), s\right) & \stackrel{(+)}{=} E\left[u(X, t) \mid \mathcal{F}_{s}\right]=E\left[E\left[u(X, t) \mid \mathcal{F}_{v}\right] \mid \mathcal{F}_{s}\right] \\
& \stackrel{(\cdot)}{=} E\left[u\left(C_{v, t}(X), v\right) \mid \mathcal{F}_{s}\right] \stackrel{(d)}{\leq} E\left[u\left(C_{v, t}(X), s\right) \mid \mathcal{F}_{s}\right] \\
& \leq u\left(E\left[C_{v, t}(X) \mid \mathcal{F}_{s}\right], s\right) .
\end{aligned}
$$

We applied in the last inequality the extended Jensen inequality, since $\left(u\left(C_{v, t}(X), s\right)\right)^{-}$is integrable. The second property follows by taking $v=t$ and observing that $C_{t, t}(X)=X$.

Remark 1.2. Comparing the definition of SDU with the existing literature about forward performances $([3,22,23])$, we may notice that the CCE does not rely on the existence of a market: this allows a higher level of generality and freedom in the choice of the preferences of the agent. If one takes into account the class of forward utilities and compute the CCE of a self-financing discounted portfolios $X^{\pi}$ (see [22] for the notations), then only for an optimal strategy $\pi_{t}^{*}$ we have that $C_{s, t}\left(X_{t}^{\pi^{*}}\right)=X_{s}^{\pi^{*}}$ whereas in general $C_{s, t}\left(X_{t}^{\pi}\right) \leq X_{s}^{\pi}$. This points out an economic interpretation of the CCE: given the final outcome of some risky position we backwardly build up a process which takes into account the agent's random riskaversion. For replicable contingent claims it means that $X_{s}^{\pi}-C_{s, t}\left(X_{t}^{\pi}\right)$ measures the gap between the real value of the claim at time $s$, and the smallest amount for which the decision maker would willingly sell the claim if he had it. The gap will be deleted whenever we move through an optimal strategy.

\section{The Right Framework for the CCE}

Until now we have considered $C_{s, t}$ as a map defined on the set of random variables $\mathcal{U}(t)$ which is not in general a vector space. In order to show the dual representation of the CCE it is convenient to define it on a Banach lattice. Therefore we now introduce the general class of Musielak-Orlicz spaces induced by the SDU taken into account. 


\subsection{Generalities on Musielak-Orlicz spaces}

Given a non-atomic probability space $(\Omega, \mathcal{F}, \mathbb{P})$ and a function $\Psi: \mathbb{R} \times \Omega \rightarrow \mathbb{R} \cup$ $\{+\infty\}$, with $\mathcal{D}=\{x \in \mathbb{R} \mid \Psi(x, \omega)<+\infty\} \neq \emptyset$, we say that $\Psi$ is a (generalized) Young function if $\Psi(x, \cdot)$ is $\mathcal{F}$-measurable and for $\mathbb{P}$ a.e. $\omega \in \Omega$

(1) $\Psi(\cdot, \omega)$ is even and convex;

(2) the effective domain $\mathcal{D}$ does not depend on $\omega$ and $0 \in \operatorname{int}(\mathcal{D})$;

(3) $\Psi(\infty, \omega)=+\infty, \Psi(0, \omega)=0$.

Note that $\Psi$ may jump to $+\infty$ outside of a bounded neighborhood of 0 . In case $\Psi$ is finite valued however, it is also continuous w.r.t. $x$ by convexity. Whenever possible, we will suppress the explicit dependence of $\Psi$ from $\omega$.

The Musielak-Orlicz space $L^{\Psi}$, on $(\Omega, \mathcal{F}, \mathbb{P})$ is then defined as

$$
L^{\Psi}=\left\{X \in L^{0} \mid \exists \alpha>0 E[\Psi(\alpha X)]<+\infty\right\} .
$$

endowed with the Luxemburg norm

$$
N_{\Psi}(X)=\inf \left\{c>0 \mid E\left[\Psi\left(X \cdot c^{-1}\right)\right] \leq 1\right\} .
$$

Although there are no particular differences with Musielak work (see [24]), here we are dropping the hypothesis on $\Psi$ to be finite (and so continuous). But since the domain $\mathcal{D}$ does not depend on $\omega$ we have that non continuous $\Psi$ s always induce the space $L^{\infty}(\Omega, \mathcal{F}, \mathbb{P})$ and the Luxemburg norm is equivalent to the supremum norm.

It is known that $\left(L^{\Psi}, N_{\Psi}\right)$ is a Banach space (Theorem 7.7 in [24]), and with the usual pointwise lattice operations, $L^{\Psi}$ is a Banach lattice.

There is an important linear subspace of $L^{\Psi}$, which is also a Banach lattice

$$
M^{\Psi}=\left\{X \in L^{0} \mid E[\Psi(\alpha X)]<+\infty \forall \alpha>0\right\} .
$$

In general, $M^{\Psi} \varsubsetneqq L^{\Psi}$ and this can be easily seen when $\Psi$ is non continuous since in this case $M^{\Psi}=\{0\}$, but there are also non trivial examples of the strict containment with finite-valued, continuous Young functions, that we will consider soon.

Other convenient assumptions on $\Psi$ that we will use in the forthcoming discussion are

(int) $E[\Psi(x)]$ is finite for every $x \in \mathcal{D}$;

(sub) there exists a Young function $g: \mathbb{R} \rightarrow \mathbb{R} \cup\{+\infty\}$ such that $g(x) \leq \Psi(x, \omega)$ for $\mathbb{P}$-a.e. $\omega \in \Omega$

$\left(\Delta_{2}\right)$ There exists $K \in \mathbb{R}, h \in L^{1}$ and $x_{0} \in \mathbb{R}$ such that

$$
\Psi(2 x, \cdot) \leq K \Psi(x, \cdot)+h(\cdot) \text { for all } x>x_{0}, \mathbb{P}-\text { a.s. }
$$

When $\Psi$ satisfies (int) and the $\left(\Delta_{2}\right)$ condition (and it is henceforth finite-valued and continuous) the two spaces $M^{\Psi}, L^{\Psi}$ coincide and $L^{\Psi}$ can simply be written as $\left\{X \in L^{0} \mid E[\Psi(X)]<+\infty\right\}$ (see [24], Theorem 8.14). This is the case of the $L^{p}$ spaces when $\Psi$ does not depend on $\omega$. 
In [24] (Theorem 7.6) it is also shown that when $\Psi$ is (int) and continuous on $\mathbb{R}$, then $M^{\Psi}=\bar{L}^{\Phi}$ with closure taken in the Luxemburg norm. When $\Psi$ is continuous but grows too quickly, it may happen that $M^{\Psi}={\overline{L^{\infty}}}^{\Psi} \varsubsetneqq L^{\Psi}$. As a consequence, simple functions are not necessarily dense in $L^{\Psi}$.

If both (int) and (sub) hold, it is not difficult to prove that

$$
L^{\infty} \hookrightarrow M^{\Psi} \hookrightarrow L^{\Psi} \hookrightarrow L^{g} \hookrightarrow L^{1}
$$

with linear lattice embeddings (the inclusions).

As usual, the convex conjugate function $\Psi^{*}$ of $\Psi$ is defined as

$$
\Psi^{*}(y, \omega)=: \sup _{x \in \mathbb{R}}\{x y-\Psi(x, \omega)\}
$$

and it is also a Young function. The function $\Psi^{*}$ in general does not satisfy (int), but a sufficient condition for it is that $\Psi$ is (sub). The Musielak-Orlicz space $L^{\Psi^{*}}$ will be endowed with the Orlicz (or dual) norm

$$
\|X\|_{\Psi^{*}}=\sup \left\{E[|X f|] \mid f \in L^{\Psi}: E[\Psi(f)] \leq 1\right\}
$$

which is equivalent to the Luxemburg norm.

\subsection{The Musielak-Orlicz space $L^{\widehat{u}}$ induced by an SDU}

In the spirit of [5], we now build the time-dependent stochastic Orlicz space induced by the $\operatorname{SDU} u(x, t, \omega)$. The even function $\widehat{u}: \mathbb{R} \times[0,+\infty) \times \Omega \rightarrow \mathbb{R} \cup\{+\infty\}$ defined by

$$
\widehat{u}(x, t, \omega)=u(0, t)-u(-|x|, t, \omega)
$$

is a Young function and the induced Orlicz spaces are

$$
\begin{aligned}
L^{\widehat{u}_{t}} & =\left\{X \in L_{\mathcal{F}_{t}}^{0} \mid \exists \alpha>0 E[\widehat{u}(\alpha X, t)]<+\infty\right\} \\
M^{\widehat{u}_{t}} & =\left\{X \in L_{\mathcal{F}_{t}}^{0} \mid E[\widehat{u}(\alpha X, t)]<+\infty \forall \alpha>0\right\}
\end{aligned}
$$

endowed with the Luxemburg norm $N_{\widehat{u}_{t}}(\cdot)$.

Notice the following important fact:

$$
M^{\widehat{u}_{t}} \subseteq \mathcal{U}(t)
$$

Indeed, for any given $\lambda>0$ and $X \in L_{\mathcal{F}_{t}}^{0}$ such that $E[\widehat{u}(\lambda X, t)]<+\infty$ we have: $E[u(\lambda X, t)] \geq E[u(-\lambda|X|, t)]>-\infty$. On the other hand $u(x, t)-u(0, t) \leq \widehat{u}(x, t)$ so that $E[u(\lambda X, t)] \leq E[\widehat{u}(\lambda X, t)+u(0, t)]<+\infty$ and the claim follows. In particular this means that (int) implies $u(x, t)$ is integrable for every $x \in \mathcal{D}(t)$.

This argument highlights one relevant feature: every $X \in M^{\widehat{u}_{t}}$ belongs to the set $\mathcal{U}(t)$ so that the CCE is well defined on $M^{\widehat{u}_{t}}$. In the following examples also $C_{s, t}(X) \in M^{\widehat{u}_{s}}$ holds true, so that $C_{s, t}: M^{\widehat{u}_{t}} \rightarrow M^{\widehat{u}_{s}}$ and it make sense to study the time consistency of $C_{s, t}$. 


\subsection{Examples}

Exponential random utilities. Let us consider $u: \mathbb{R} \times[0, \infty) \times \Omega \rightarrow \mathbb{R}$ defined by

$$
u(x, t, \omega)=-e^{-\alpha_{t}(\omega) x+\beta_{t}(\omega)}
$$

where $\alpha_{t}>0$ and $\beta_{t}$ are adapted stochastic processes.

In this example the CCE may be simply computed inverting the function $u(\cdot, t, \omega)$ :

$$
C_{s, t}(X)=-\frac{1}{\alpha_{s}} \ln \left\{E\left[e^{-\alpha_{t} X+\beta_{t}} \mid \mathcal{F}_{s}\right]\right\}+\frac{\beta_{s}}{\alpha_{s}}
$$

Notice the measurability requirement on the risk aversion process $\alpha_{t}$, which is different from what can be found in some examples in the literature related to dynamic risk measures, as e.g. in [1], where the $\alpha_{t}$ in (2.1) is replaced by $\alpha_{s}$.

Assumptions. We suppose that $\beta_{t}$ belongs to $L^{\infty}\left(\mathcal{F}_{t}\right)$ for any $t>0$ and that $e^{\alpha_{t} x} \in L_{\mathcal{F}_{t}}^{1}$ for every $x \in \mathbb{R}$.

These assumptions guarantee that (int) holds. In particular if $\alpha_{t}(\omega) \equiv \alpha \in \mathbb{R}$ and $\beta_{t} \equiv 0$ then $C_{s, t}(X)=-\rho_{s, t}(X)$, where $\rho_{s, t}$ is the dynamic entropic risk measure induced by the exponential utility. Unfortunately when the risk aversion coefficient is stochastic we have no chance that $C_{s, t}$ has any monetary property. On the other hand monotonicity and concavity keep standing. The first is due to Proposition 1.1, whereas the second is a straightforward application of Holderconditional inequality. This means that in general $\rho_{s, t}(X)=:-C_{s, t}(X)$ satisfies all the usual assumptions of dynamic risk measures, only failing the cash additive property. We now show a sufficient condition by which $\rho_{s, t}(X)$ is at least cash subadditive, i.e. $\rho_{s, t}(X+Y) \geq \rho_{s, t}(X)-Y$ where $Y \in L_{\mathcal{F}_{s}}^{\infty}$ and $Y \geq 0$.

Proposition 2.1. Under the previous assumptions, the functional

$$
\rho_{s, t}(X)=\frac{1}{\alpha_{s}} \ln \left\{E\left[e^{-\alpha_{t} X+\beta_{t}} \mid \mathcal{F}_{s}\right]\right\}+\frac{\beta_{s}}{\alpha_{s}}
$$

is cash subadditive if the process $\left\{\alpha_{t}\right\}_{t \geq 0}$ is almost surely decreasing.

Proof. For every $Y \in L_{\mathcal{F}_{s}}^{\infty}$ and $Y \geq 0$ :

$$
\begin{aligned}
\rho_{s, t}(X+Y) & =\frac{1}{\alpha_{s}} \ln \left\{E\left[e^{-\frac{\alpha_{t}}{\alpha_{s}} \alpha_{s} Y} e^{-\alpha_{t} X+\beta_{t}} \mid \mathcal{F}_{s}\right]\right\}-\frac{\beta_{s}}{\alpha_{s}} \\
& \geq \frac{1}{\alpha_{s}} \ln \left\{E\left[e^{-\alpha_{s} Y} e^{-\alpha_{t} X+\beta_{t}} \mid \mathcal{F}_{s}\right]\right\}-\frac{\beta_{s}}{\alpha_{s}}=\rho_{s, t}(X)-Y .
\end{aligned}
$$

Proposition 2.2. Under the the previous assumptions

$$
C_{s, t}: M^{\widehat{u}_{t}} \rightarrow M^{\widehat{u}_{s}}
$$


Proof. Let $\lambda \geq 1$, and since no confusion arises we denote by $u_{t}(x) \stackrel{\circ}{=} u(x, t)$. Define $A=\left\{\ln E\left[e^{-\alpha_{t} X+\beta_{t}} \mid \mathcal{F}_{s}\right] \leq \beta_{s}\right\}$ and notice that

$$
\begin{aligned}
\widehat{u}_{s}\left(\lambda C_{s, t}(X)\right)-u_{s}(0)=-u_{s}\left(-\frac{\lambda}{\alpha_{s}}\left|-\ln E\left[e^{-\alpha_{t} X+\beta_{t}} \mid \mathcal{F}_{s}\right]+\beta_{s}\right|\right) \\
=e^{\beta_{s}} \exp \left(\lambda\left|-\ln E\left[e^{-\alpha_{t} X+\beta_{t}} \mid \mathcal{F}_{s}\right]+\beta_{s}\right|\right) \\
=e^{\beta_{s}} \exp \left(\lambda\left(\beta_{s}-\ln E\left[e^{-\alpha_{t} X+\beta_{t}} \mid \mathcal{F}_{s}\right]\right)\right) \mathbf{1}_{A} \\
\quad+e^{\beta_{s}} \exp \left(\lambda\left(\ln E\left[e^{-\alpha_{t} X+\beta_{t}} \mid \mathcal{F}_{s}\right]-\beta_{s}\right)\right) \mathbf{1}_{A^{C}} \\
=e^{\beta_{s}(1+\lambda)} E\left[e^{-\alpha_{t} X+\beta_{t}} \mid \mathcal{F}_{s}\right]^{-\lambda} \mathbf{1}_{A}+e^{\beta_{s}(1-\lambda)} E\left[e^{-\alpha_{t} X+\beta_{t}} \mid \mathcal{F}_{s}\right]^{\lambda} \mathbf{1}_{A^{C}}
\end{aligned}
$$

Since on $A$ we have $E\left[e^{-\alpha_{t} X+\beta_{t}} \mid \mathcal{F}_{s}\right] \leq e^{\beta_{s}}$ and in general $e^{\beta_{s}(1-\lambda)} \leq a \in \mathbb{R}_{+}$then

$$
\begin{aligned}
E\left[\widehat{u}_{s}\left(\lambda C_{s, t}(X)\right)\right] & \leq E\left[e^{\beta_{s}(1+\lambda-\lambda)} \mathbf{1}_{A}\right]+a E\left\{E\left[e^{-\alpha_{t} X+\beta_{t}} \mid \mathcal{F}_{s}\right]^{\lambda} \mathbf{1}_{A^{C}}\right\}+E\left[u_{s}(0)\right] \\
& \leq-E\left[u_{s}(0)\right]+a E\left[e^{\lambda\left(-\alpha_{t} X+\beta_{t}\right)}\right]+E\left[u_{s}(0)\right] \\
& \leq+a\left\|\left(e^{(\lambda-1) \beta_{t}}\right)\right\|_{\infty} E\left[\widehat{u}_{t}(\lambda X)+e^{\beta_{t}}\right] \leq K E\left[\widehat{u}_{t}(\lambda X)\right]
\end{aligned}
$$

Notice that the second step is a simple application of Jensen's inequality, in fact: $E[Y \mid \mathcal{G}]^{\lambda} \leq E\left[Y^{\lambda} \mid \mathcal{G}\right] \forall \lambda \geq 1$. Moreover we have that for $0<\lambda<1$ $E\left[\widehat{u}_{s}\left(\lambda C_{s, t}(X)\right)\right] \leq E\left[\widehat{u}_{s}\left(C_{s, t}(X)\right)\right]<\infty$ and then $C_{s, t}(X) \in M^{\widehat{u}_{s}}$.

Random-power utility. Consider the utility function given by

$$
u(x, t, \omega)=-\gamma_{t}(\omega)|x|^{p_{t}(\omega)} \mathbf{1}_{(-\infty, 0)}
$$

where $\gamma_{t}, p_{t}$ are adapted stochastic processes satisfying $\gamma_{t}>0$ and $p_{t}>1$. We have $\widehat{u}(x, t)=\gamma_{t}|x|^{p_{t}}$. Here assumption (int) is troublesome but not needed for what follows. On the other hand the utility fails to be strictly increasing so that we won't have uniqueness of the solution for the equation defining the CCE, namely

$$
-\gamma_{s}\left|C_{s, t}(X)\right|^{p_{s}} \mathbf{1}_{\left\{C_{s, t}(X)<0\right\}}=E\left[-\gamma_{t}|X|^{p_{t}} \mathbf{1}_{\{X<0\}} \mid \mathcal{F}_{s}\right]
$$

Notice that $C_{s, t}(X)=C_{s, t}\left(X^{-}+K \mathbf{1}_{X \geq 0}\right)$ where $K$ is any positive $\mathcal{F}_{t}$ r.v.; moreover if $G:=\left\{E\left[\gamma_{t}|X|^{p_{t}} \mathbf{1}_{\{X<0\}} \mid \mathcal{F}_{s}\right]>0\right\}$ then $\mathbb{P}\left(G \backslash\left\{C_{s, t}(X)<0\right\}\right)=0$. If we decompose $X$ as $X^{+}-X^{-}$we can conclude that

$$
C_{s, t}(X)=-\frac{1}{\gamma_{s}}\left(E\left[\gamma_{t}\left(X^{-}\right)^{p_{t}} \mid \mathcal{F}_{s}\right]\right)^{\frac{1}{p_{s}}}+K \mathbf{1}_{G^{C}}
$$

it's the class of solutions of $(2.2)$ where $K \in L_{\mathcal{F}_{s}}^{0}$ and $K>0$. This is a natural consequence of the choice of a preference system in which the agent is indifferent among all the positive variables. If in particular $K \in M^{\hat{u}_{s}}$ then it is easy to check that $C_{s, t}: M^{\widehat{u}_{t}} \rightarrow M^{\widehat{u}_{s}}$. 
A general result. One may wonder what happens for an arbitrary SDU. Clearly the fact that $C_{s, t}$ is a map between the the two corresponding Orlicz spaces at time $t$ and $s$ is a key feature for the time-consistency. We take into account a particular class of SDU, which are a stochastic transformation of a standard utility function.

Let $V: \mathbb{R} \rightarrow \mathbb{R}$ a concave, strictly increasing function: take an adapted stochastic process, $\left\{\alpha_{t}\right\}_{t \geq 0}$, such that for every $t \geq 0, \alpha_{t}>0$. Then $u(x, t, \omega)=V\left(\alpha_{t}(\omega) x\right)$ is a SDU and

$$
C_{s, t}(X)=\frac{1}{\alpha_{s}} V^{-1}\left(E\left[V\left(\alpha_{t} X\right) \mid \mathcal{F}_{s}\right]\right)
$$

Proposition 2.3. Let $\Theta_{t}=\left\{X \in L^{\widehat{u}_{t}} \mid E\left[u\left(-X^{-}, t\right)\right]>-\infty\right\} \supseteq M^{\widehat{u}_{t}}$. Then

$$
C_{s, t}: \Theta_{t} \rightarrow \Theta_{s}
$$

Moreover if $\widehat{u}(x, s)$ satisfies the $\left(\Delta_{2}\right)$ condition, then

$$
C_{s, t}: M^{\widehat{u}_{t}} \rightarrow M^{\widehat{u}_{s}} .
$$

Proof. Denote $\widehat{u}_{t}(x)=\widehat{u}(x, t)$; from Jensen inequality we have

$$
\frac{1}{\alpha_{s}} V^{-1}\left(E\left[V\left(\alpha_{t} X\right) \mid \mathcal{F}_{s}\right]\right) \leq \frac{1}{\alpha_{s}} E\left[\alpha_{t} X \mid \mathcal{F}_{s}\right]
$$

Define the $\mathcal{F}_{s}$ measurable sets

$$
F=\left\{E\left[V\left(\alpha_{t} X\right) \mid \mathcal{F}_{s}\right] \geq V(0)\right\}, \quad G=\left\{E\left[\alpha_{t} X \mid \mathcal{F}_{s}\right] \geq 0\right\}
$$

and deduce from equation (2.3) that

$$
0 \leq C_{s, t}(X)^{+}=\frac{1}{\alpha_{s}} V^{-1}\left(E\left[V\left(\alpha_{t} X\right) \mid \mathcal{F}_{s}\right]\right) \mathbf{1}_{F} \leq \frac{1}{\alpha_{s}} E\left[\left(\alpha_{t} X\right) \mathbf{1}_{G} \mid \mathcal{F}_{s}\right]
$$

For every $X \in L^{\widehat{u}_{t}}$ we may find a $\lambda>0$ such that $E\left[\hat{u}_{t}\left(\lambda X \mathbf{1}_{G}\right)\right]<+\infty$ :

$$
\begin{aligned}
& E\left[\hat{u}_{s}\left(\frac{\lambda}{\alpha_{s}} V^{-1}\left(E\left[V\left(\alpha_{t} X\right) \mid \mathcal{F}_{s}\right]\right) \mathbf{1}_{F}\right)\right] \\
& \quad \leq E\left[\hat{u}_{s}\left(\frac{\lambda}{\alpha_{s}} E\left[\left(\alpha_{t} X\right) \mathbf{1}_{G} \mid \mathcal{F}_{s}\right]\right)\right] \\
& \quad=E\left[V(0)-V\left(-E\left[\left(\lambda \alpha_{t} X\right) \mathbf{1}_{G} \mid \mathcal{F}_{s}\right]\right)\right] \\
& \quad \leq E\left[V(0)-V\left(-\lambda \alpha_{t} X \mathbf{1}_{G}\right)\right] \leq E\left[\widehat{u}_{t}\left(\lambda X \mathbf{1}_{G}\right)\right] .
\end{aligned}
$$

Hence $X \in L^{\widehat{u}_{t}}$ implies $C_{s, t}(X)^{+} \in L^{\widehat{u}_{s}}$.

Now let's consider a r.v. $X \in \Theta_{t}:-C_{s, t}(X)^{-}=\frac{1}{\alpha_{s}} V^{-1}\left(E\left[V\left(\alpha_{t} X\right) \mid \mathcal{F}_{s}\right]\right) \mathbf{1}_{F^{C}}$.

We can conclude that

$$
\begin{aligned}
0 \leq E\left[\widehat{u}_{s}\left(-C_{s, t}(X)^{-}\right)\right] & =E\left[-V \circ V^{-1}\left(E\left[V\left(\alpha_{t} X\right) \mathbf{1}_{F^{C}} \mid \mathcal{F}_{s}\right]\right)+V(0)\right] \\
& =E\left[-V\left(\alpha_{t} X\right) \mathbf{1}_{F^{C}}+V(0)\right]<+\infty
\end{aligned}
$$


where the last inequality follows from $X \in \Theta_{t},\{X \geq 0\} \subseteq F$ and

$$
\begin{aligned}
V\left(\alpha_{t} X\right) \mathbf{1}_{F^{C}} & =\left(V\left(\alpha_{t} X^{+}\right) \mathbf{1}_{\{X \geq 0\}}+V\left(-\alpha_{t} X^{-}\right) \mathbf{1}_{\{X<0\}}\right) \mathbf{1}_{F^{C}} \\
& =V\left(-\alpha_{t} X^{-}\right) \mathbf{1}_{\{X<0\} \cap F^{C}}
\end{aligned}
$$

This shows that surely $C_{s, t}(X) \in \Theta_{s}$, if $X \in \Theta_{t}$.

\section{Dual Representation of CCE}

In this section we prove a dual formula for the CCE, which is similar to the general result that can be found in [13]: due to the particular structure of the CCE the proof is simpler and more readable.

Consider the condition:

$$
\text { there exists } X^{*} \in\left(L^{\widehat{u}_{t}}\right)^{*} \text { s.t. } E\left[f^{*}\left(X^{*}, t\right)\right]<+\infty
$$

where $f^{*}(x, t, \omega)=\sup _{y \in \mathbb{R}}\{x y+u(y, t, \omega)\}$.

As a consequence of Theorem 1 [26], we may deduce that if (3.1) holds, if $\widehat{u}(x, t)$ is (int) and $X \in L^{\widehat{u}_{t}}$ then $E[u(\lambda X, t)]<+\infty$ for every $\lambda>0$.

Remark 3.1. The condition (3.1) is quite weak: it is satisfied, for example, if $u(x, t, \omega) \leq a x+b$ with $a, b \in \mathbb{R}$ since

$$
f^{*}(-a, t, \omega) \leq \sup _{y \in \mathbb{R}}\{(-a+a) y+b\}=b .
$$

We now take into account $\left(L^{\Psi}\right)^{*}$, the norm dual of $L^{\Psi}$ and consider the following three cases which cover a pretty large class of possible Young functions.

(1) $\Psi(\cdot, \omega)$ is (int) and discontinuous, i.e. $\mathcal{D} \varsubsetneqq \mathbb{R}$.

In this case, $L^{\Psi}=L^{\infty}$ and from the Yosida-Hewitt decomposition for elements of $b a(\Omega, \mathcal{F}, P)$ we have

$$
b a=\left(L^{\infty}\right)^{*}=L^{1} \oplus \mathcal{A}^{d},
$$

where $\mathcal{A}^{d}$ consists of pure charges, i.e. purely finitely additive measures (which are not order continuous).

(2) $\Psi(\cdot, \omega)$ is continuous, $\Psi$ and $\Psi^{*}$ are (int) and satisfy:

$$
\frac{\Psi(x, \omega)}{x} \rightarrow+\infty \mathbb{P}-a . s, \quad \text { as } x \rightarrow \infty .
$$

These conditions are not restrictive and hold as soon as $\Psi$ is (int) and (sub) with $\lim _{x \rightarrow \infty} \frac{g(x)}{x} \rightarrow+\infty$. For such Young functions it can be easily deduced from Theorem 13.17 in [24] that $\left(M^{\Psi}\right)^{*}=L^{\Psi^{*}}: \mu_{r} \in\left(M^{\Psi}\right)^{*}$ can be identified with its density $\frac{d \mu_{r}}{d P} \in L^{\Psi^{*}}$ so that we will write its action on $X \in L^{\Psi}$ as $\mu_{r}(X)=E\left[\mu_{r} X\right]$. Moreover $\left(M^{\Psi}\right)^{*}$ is a band in the dual space $\left(L^{\Psi}\right)^{*}$ (see $[2]$ Sec. 8) so that we may decompose

$$
\left(L^{\Psi}\right)^{*}=\left(M^{\Psi}\right)^{*} \oplus\left(M^{\Psi}\right)^{\perp}
$$


i.e. every $X^{*} \in\left(L^{\Psi}\right)^{*}$ can be uniquely represented as $X^{*}=\mu_{r}+\mu_{s}$ where $\mu_{s}$ belongs to the annihilator of $M^{\Psi}\left(\mu_{s}(X)=0\right.$ for every $\left.X \in M^{\Psi}\right)$ and $\mu_{r} \in\left(M^{\Psi}\right)^{*}=L^{\Psi^{*}}$. Notice that every element $\mu_{r} \in\left(M^{\Psi}\right)^{*}$ is clearly order continuous. Moreover it can be shown, applying an argument similar to the one used in Lemma $10[5]$, that every $\mu_{s} \in\left(M^{\Psi}\right)^{\perp}$ is not order continuous.

(3) $\Psi(\cdot, \omega)$ is continuous and

$$
0<a=\text { ess } \inf _{\omega \in \Omega} \lim _{x \rightarrow \infty} \frac{\Psi(x, \omega)}{x} \leq \text { ess } \sup _{\omega \in \Omega} \lim _{x \rightarrow \infty} \frac{\Psi(x, \omega)}{x}=b<+\infty
$$

Here (int) automatically holds for both $\Psi$ and $\Psi^{*}$. It follows that $L^{\Psi}=L^{1}$ and the $L^{1}$-norm is equivalent to the Luxemburg norm, so that $\left(L^{\Psi}\right)^{*}=L^{\Psi^{*}}=L^{\infty}$.

Assumptions for the dual result. In this section $u(x, t, \omega)$ is a SDU, such that:

(1) For all $t \geq 0$, the induced Young function $\widehat{u}(x, t, \omega)$ belongs to one of the three classes mentioned above

(2) The condition (3.1) holds true.

As shown above, under the assumption (1) the order dual space of $L^{\widehat{u}_{t}}$ is known and is contained in $L^{1}$. This will also allow us to apply Proposition A.1. The second assumption implies that $E[u(\cdot, t)]: L^{\widehat{u}_{t}} \rightarrow[-\infty,+\infty)$ is a well defined convex functional ([26]).

Thus we have $u\left(X^{+}, t\right) \in L_{\mathcal{F}_{t}}^{1}$, but in general we do not have integrability for $u\left(-X^{-}, t\right)$. This means that if $X \notin \Theta_{t}=\left\{X \in L^{\widehat{u}_{t}} \mid E\left[u\left(-X^{-}, t\right)\right]>-\infty\right\}$ we are forced to consider the generalized conditional expectation

$$
E\left[u(X, t) \mid \mathcal{F}_{s}\right]:=E\left[u(X, t)^{+} \mid \mathcal{F}_{s}\right]-\lim _{n} E\left[u(X, t)^{-} \mathbf{1}_{\left\{-n \leq-u(X, s)^{-}<0\right\}} \mid \mathcal{F}_{s}\right],
$$

which can be equivalently written as:

$$
E\left[u(X, t) \mid \mathcal{F}_{s}\right]=E\left[u\left(X^{+}, t\right) \mathbf{1}_{\{X \geq 0\}} \mid \mathcal{F}_{s}\right]+\lim _{n} E\left[u\left(-X^{-}, t\right) \mathbf{1}_{\{-n \leq X<0\}} \mid \mathcal{F}_{s}\right] .
$$

Therefore, $E\left[u(X, t) \mid \mathcal{F}_{s}\right] \in \bar{L}_{\mathcal{F}_{s}}^{0}$ and $C_{s, t}(\cdot)$ is defined on the entire space $L^{\widehat{u}_{t}}$. We fix throughout this section $0<s \leq t$ and define

$$
\begin{gathered}
\mathcal{P}_{\mathcal{F}_{t}}=\left\{X^{*} \in\left(L^{\widehat{u}_{t}^{*}}\right)_{+} \mid E\left[X^{*}\right]=1\right\} \subseteq\{Q \ll \mathbb{P} \mid Q \text { probability }\} \\
U: L^{\widehat{u}_{t}} \rightarrow \bar{L}_{\mathcal{F}_{s}}^{0} \text { given by } U(X):=E\left[u(X, t) \mid \mathcal{F}_{s}\right]
\end{gathered}
$$

The map $U$ is concave and increasing and admits the dual representation stated in Lemma 3.1. From equation (3.3) we deduce the dual representation of $C_{s, t}(\cdot)=$ $u^{-1}(U(\cdot), s)$ as follows.

Theorem 3.1. Fix $s \leq t$. For every $X \in L^{\widehat{u}_{t}}$

$$
C_{s, t}(X)=\inf _{Q \in \mathcal{P}_{\mathcal{F}_{t}}} G\left(E_{Q}\left[X \mid \mathcal{F}_{s}\right], Q\right)
$$


where for every $Y \in L_{\mathcal{F}_{s}}^{0}$,

$$
G(Y, Q)=\sup _{\xi \in L^{\widehat{u}_{t}}}\left\{C_{s, t}(\xi) \mid E_{Q}\left[\xi \mid \mathcal{F}_{s}\right]={ }_{Q} Y\right\}
$$

Moreover if $X \in M^{\widehat{u}_{t}}$ then the essential infimum in (3.2) is actually a minimum.

The proof is based on the following lemma.

Lemma 3.1. Let $s \leq t$. For every $X \in L^{\widehat{u}_{t}}$

$$
U(X)=\inf _{Q \in \mathcal{P}_{\mathcal{F}_{t}}} S\left(E_{Q}\left[X \mid \mathcal{F}_{s}\right], Q\right)
$$

where $S(Y, Q)=\sup _{\xi \in L^{u_{t}}}\left\{U(\xi) \mid E_{Q}\left[\xi \mid \mathcal{F}_{s}\right]={ }_{Q} Y\right\}$ for any $Y \in L_{\mathcal{F}_{s}}^{0}$.

Moreover if $X \in M^{\widehat{u}_{t}}$ then the essential infimum in (3.3) is actually a minimum.

Proof. Obviously $\forall Q \in \mathcal{P}_{\mathcal{F}_{t}}$

$$
E\left[u(X, t) \mid \mathcal{F}_{s}\right] \leq \sup _{\xi \in L^{\hat{u}_{t}}}\left\{U(\xi) \mid E_{Q}\left[\xi \mid \mathcal{F}_{s}\right]={ }_{Q} E_{Q}\left[X \mid \mathcal{F}_{s}\right]\right\}
$$

and then

$$
E\left[u(X, t) \mid \mathcal{F}_{s}\right] \leq \inf _{Q \in \mathcal{P}_{\mathcal{F}_{t}}} \sup _{\xi \in L^{\widehat{u}_{t}}}\left\{U(\xi) \mid E_{Q}\left[\xi \mid \mathcal{F}_{s}\right]={ }_{Q} E_{Q}\left[X \mid \mathcal{F}_{s}\right]\right\}
$$

Important remark: we have that $E(U(X))=E(u(X, t))$; this means that

$$
E(U(\cdot)): L^{\widehat{u}_{t}} \rightarrow[-\infty,+\infty)
$$

is a concave functional. From the monotone convergence theorem and Jensen inequality the functional $E(u(X, t))$ is continuous from above (i.e. $X_{n} \downarrow X \Rightarrow$ $\left.E\left(u\left(X_{n}, t\right)\right) \downarrow E(u(X, t))\right)$. Applying Lemma 15 in [6], $E(U(X))$ is order u.s.c. and thus $\sigma\left(L^{\widehat{u}_{t}}, L^{\widehat{u}_{t}^{*}}\right)$-u.s.c. (Proposition $24[6]$ ).

From Proposition A.1 in the Appendix:

$$
\begin{aligned}
E(U(X)) & =\inf _{Q \in \mathcal{P}_{\mathcal{F}_{t}}} \sup _{\xi \in L^{\widehat{u}_{t}}}\left\{E[U(\xi)] \mid E_{Q}[\xi]=E_{Q}[X]\right\} \\
& \geq \inf _{Q \in \mathcal{P}_{\mathcal{F}_{t}}} \sup _{\xi \in L^{\widehat{u}_{t}}}\left\{E(U(\xi)) \mid E_{Q}\left[\xi \mid \mathcal{F}_{s}\right]={ }_{Q} E_{Q}\left[X \mid \mathcal{F}_{s}\right]\right\} \geq E(U(X))
\end{aligned}
$$

i.e.

$$
E(U(X))=\inf _{Q \in \mathcal{P}_{\mathcal{F}_{t}}} \sup _{\xi \in L^{\widehat{u}_{t}}}\left\{E(U(\xi)) \mid E_{Q}\left[\xi \mid \mathcal{F}_{s}\right]={ }_{Q} E_{Q}\left[X \mid \mathcal{F}_{s}\right]\right\}
$$

Surely the map $U$ is regular (i.e. for every $A \in \mathcal{F}_{s}, U\left(X \mathbf{1}_{A}+Y \mathbf{1}_{A^{C}}\right)=U(X) \mathbf{1}_{A}+$ $\left.U(Y) \mathbf{1}_{A^{C}}\right)$ and then the set $\mathcal{A}=\left\{U(\xi) \mid E_{Q}\left[\xi \mid \mathcal{F}_{s}\right]={ }_{Q} E_{Q}\left[X \mid \mathcal{F}_{s}\right]\right\}$ is upward directed. In fact given $\xi_{1}, \xi_{2} \in \mathcal{A}$ we have

$$
U\left(\xi_{1}\right) \vee U\left(\xi_{2}\right)=U\left(\xi_{1}\right) \mathbf{1}_{F}+U\left(\xi_{2}\right) \mathbf{1}_{F^{C}}=U\left(\xi_{1} \mathbf{1}_{F}+\xi_{2} \mathbf{1}_{F^{C}}\right)
$$


where $F=\left\{U\left(\xi_{1}\right) \geq U\left(\xi_{2}\right)\right\}$ and $E_{Q}\left[\xi_{1} \mathbf{1}_{F}+\xi_{2} \mathbf{1}_{F^{C}} \mid \mathcal{F}_{s}\right]={ }_{Q} E_{Q}\left[X \mid \mathcal{F}_{s}\right]$. By this last property and the monotone convergence theorem we deduce

$$
E\left[S\left(E_{Q}\left[X \mid \mathcal{F}_{s}\right], Q\right)\right]=\sup _{\xi \in L^{\hat{u}_{t}}}\left\{E[U(\xi)] \mid E_{Q}\left[\xi \mid \mathcal{F}_{s}\right]={ }_{Q} E_{Q}\left[X \mid \mathcal{F}_{s}\right]\right\}
$$

Hence

$$
\begin{aligned}
E(U(X)) & =\inf _{Q \in \mathcal{P}_{\mathcal{F}_{t}}} \sup _{\xi \in L^{\hat{u}_{t}}}\left\{E(U(\xi)) \mid E_{Q}\left[\xi \mid \mathcal{F}_{s}\right]={ }_{Q} E_{Q}\left[X \mid \mathcal{F}_{s}\right]\right\} \\
& =\inf _{Q \in \mathcal{P}_{\mathcal{F}_{t}}} E\left(\sup _{\xi \in L^{\hat{u}_{t}}}\left\{U(\xi) \mid E_{Q}\left[\xi \mid \mathcal{F}_{s}\right]={ }_{Q} E_{Q}\left[X \mid \mathcal{F}_{s}\right]\right\}\right) \\
& \geq E\left(\inf _{Q \in \mathcal{P}_{\mathcal{F}_{t}}} \sup _{\xi \in L^{\hat{u}_{t}}}\left\{U(\xi) \mid E_{Q}\left[\xi \mid \mathcal{F}_{s}\right]={ }_{Q} E_{Q}\left[X \mid \mathcal{F}_{s}\right]\right\}\right)
\end{aligned}
$$

This last chain of inequalities together with inequality (3.4) gives

$$
U(X)=\inf _{Q \in \mathcal{P}_{\mathcal{F}_{t}}} \sup _{\xi \in L^{\hat{u}_{t}}}\left\{U(\xi) \mid E_{Q}\left[\xi \mid \mathcal{F}_{s}\right]={ }_{Q} E_{Q}\left[X \mid \mathcal{F}_{s}\right]\right\} \quad \forall X \in L^{\widehat{u}_{t}}
$$

Moreover from generalized Namioka-Klee theorem, the functional $E(u(\cdot)): L^{\widehat{u}_{t}}$ is norm continuous on $\operatorname{int}\left(\Theta_{u}\right) \supseteq M^{\widehat{u}_{t}}$ (see [6] Lemma 32) and then $E(U(X))$ as well since $E(U(X))=E(u(X))$.

From Proposition A.1 we have that:

$$
\begin{aligned}
E(U(X)) & =\min _{Q \in \mathcal{P}_{\mathcal{F}_{t}}} \sup _{\xi \in M^{\widehat{u}_{t}}}\left\{E(U(\xi)) \mid E_{Q}[\xi]=E_{Q}[X]\right\} \\
& =\sup _{\xi \in M^{\widehat{u}_{t}}}\left\{E[U(\xi)] \mid E_{Q_{\min }}[\xi]=E_{Q_{\min }}[X]\right\} \\
& \geq \sup _{\xi \in M^{\hat{u}_{t}}}\left\{E[U(\xi)] \mid E_{Q_{\min }}\left[\xi \mid \mathcal{F}_{s}\right]=Q_{\min } E_{Q_{\min }}\left[X \mid \mathcal{F}_{s}\right]\right\} \geq E[(U(X))
\end{aligned}
$$

The remaining proof matches the previous case and then we get

$$
U(X)=\min _{Q \in \mathcal{P}_{\mathcal{F}_{t}}} \sup _{\xi \in M^{\widehat{u}_{t}}}\left\{U(\xi) \mid E_{Q}\left[\xi \mid \mathcal{F}_{s}\right]={ }_{Q} E_{Q}\left[X \mid \mathcal{F}_{s}\right]\right\} \quad \forall X \in M^{\widehat{u}_{t}}
$$

where the minimizer is exactly $Q_{\min }$.

Proof. [of Theorem 3.1] Since $s, t$ are fixed throughout this proof we redefine $C_{s, t}(\cdot)=C(\cdot), u(x, t)=u(x)$ and $u(x, s)=v(x)$. We show that for every fixed $Q \in \mathcal{P}_{\mathcal{F}_{t}}, v^{-1} S\left(E_{Q}\left[X \mid \mathcal{F}_{s}\right], Q\right)=G\left(E_{Q}\left[X \mid \mathcal{F}_{s}\right], Q\right)$.

Since $C, U$ are regular, for every fixed $Q \in \mathcal{P}_{\mathcal{F}}$ the sets

$$
\begin{aligned}
& \left\{C(\xi) \mid \xi \in L^{\widehat{u}_{t}}, E_{Q}\left[\xi \mid \mathcal{F}_{s}\right]={ }_{Q} E_{Q}\left[X \mid \mathcal{F}_{s}\right]\right\}, \\
& \left\{U(\xi) \mid \xi \in L^{\widehat{u}_{t}}, E_{Q}\left[\xi \mid \mathcal{F}_{s}\right]={ }_{Q} E_{Q}\left[X \mid \mathcal{F}_{s}\right]\right\}
\end{aligned}
$$


are upward directed and then there exist $\xi_{h}^{Q}, \eta_{h}^{Q}$ such that $E_{Q}\left[\xi_{h}^{Q} \mid \mathcal{F}_{s}\right]={ }_{Q}$ $E_{Q}\left[X \mid \mathcal{F}_{s}\right], E_{Q}\left[\eta_{h}^{Q} \mid \mathcal{F}_{s}\right]={ }_{Q} E_{Q}\left[X \mid \mathcal{F}_{s}\right]$, for every $h>0$, and

$$
C\left(\xi_{h}^{Q}\right) \uparrow G\left(E_{Q}\left[X \mid \mathcal{F}_{s}\right], Q\right), \quad U\left(\eta_{h}^{Q}\right) \uparrow S\left(E_{Q}\left[X \mid \mathcal{F}_{s}\right], Q\right) \quad \mathbb{P} \text {-a.s. }
$$

Thus since $v^{-1}$ is continuous in the interior of its domain:

$$
\begin{aligned}
G\left(E_{Q}\left[X \mid \mathcal{F}_{s}\right], Q\right) & \geq \lim _{h} C\left(\eta_{h}^{Q}\right)=v^{-1} \lim _{h} U\left(\eta_{h}^{Q}\right)=v^{-1} S\left(E_{Q}\left[X \mid \mathcal{F}_{s}\right], Q\right) \\
& \geq v^{-1} \lim _{h} U\left(\xi_{h}^{Q}\right)=\lim _{h} C\left(\xi_{h}^{Q}\right)=G\left(E_{Q}\left[X \mid \mathcal{F}_{s}\right], Q\right)
\end{aligned}
$$

and this ends the first claim.

It's not hard to prove that the infimum is actually a limit (using the property of downward directness of the set as has been shown in Lemma 19 (iv) in [13]): therefore we deduce from the continuity of $v^{-1}$ that

$$
\begin{aligned}
C(X) & =v^{-1} \inf _{Q \in \mathcal{P}_{\mathcal{F}_{t}}} S\left(E_{Q}\left[X \mid \mathcal{F}_{s}\right], Q\right) \\
& =\inf _{Q \in \mathcal{P}_{\mathcal{F}_{t}}} v^{-1} S\left(E_{Q}\left[X \mid \mathcal{F}_{s}\right], Q\right)=\inf _{Q \in \mathcal{P}_{\mathcal{F}_{t}}} G\left(E_{Q}\left[X \mid \mathcal{F}_{s}\right], Q\right) .
\end{aligned}
$$

\section{Appendix}

\section{A.1. On quasiconvex real valued maps}

Let $L$ be a locally convex topological vector space, $L^{*}$ be its dual space and $f: L \rightarrow$ $\overline{\mathbb{R}}:=\mathbb{R} \cup\{-\infty\} \cup\{\infty\}$. Define: $R: L^{*} \times \mathbb{R} \rightarrow \overline{\mathbb{R}}$ by

$$
R\left(X^{*}, t\right):=\sup \left\{f(X) \mid X \in L \text { such that } X^{*}(X) \geq t\right\} .
$$

Theorem A.1. Let $f$ as before

(i) If $f$ is lsc then: $f(X)=\sup _{X^{*} \in L^{*}} R\left(X^{*}, X^{*}(X)\right)$.

(ii) If $f$ is usc then: $f(X)=\max _{X^{*} \in L^{*}} R\left(X^{*}, X^{*}(X)\right)$.

Proof. By definition, for any $X^{*} \in L^{*}, R\left(X^{*}, X^{*}(X)\right) \leq f(X)$ and therefore

$$
\sup _{X^{*} \in L^{*}} R\left(X^{*}, X^{*}(X)\right) \leq f(X), \quad X \in L .
$$

(i) See [13] Theorem 1.1.

(ii) For any fixed $X \in L$, the set $\{\xi \in L: f(\xi)<f(X)\}:=\mathcal{E}$ is convex open and $X \notin \mathcal{E}$. By the Hahn Banach theorem there exists a continuous linear functional that properly separates $X$ and $\mathcal{E}$, i.e. there exists $\alpha \in \mathbb{R}$ and $X^{*} \in L^{*}$ such that: $X^{*}(X)>\alpha \geq X^{*}(\xi)$ for all $\xi \in \mathcal{E}$.

Hence: $\left\{\xi \in L: X^{*}(\xi) \geq X^{*}(X)\right\} \subseteq(\mathcal{E})^{C}=\{\xi \in L: f(\xi) \geq f(X)\}$ and

$$
\begin{aligned}
f(X) & \geq \sup _{Y^{*} \in L^{*}} R\left(Y^{*}, Y^{*}(X)\right) \geq R\left(X^{*}, X^{*}(X)\right) \\
& =\inf \left\{f(\xi) \mid \xi \in L \text { such that } X^{*}(\xi) \geq X^{*}(X)\right\} \\
& \geq \inf \left\{f(\xi) \mid \xi \in(\mathcal{E})^{C}\right\} \geq f(X) .
\end{aligned}
$$


Proposition A.1. Suppose $L$ is a lattice, $L^{*}=(L, \geq)^{*}$ is the order continuous dual space satisfying $L^{*} \hookrightarrow L^{1}$ and $\left(L, \sigma\left(L, L^{*}\right)\right)$ is a locally convex TVS. If $f: L \rightarrow \overline{\mathbb{R}}$ is quasiconvex, $\sigma\left(L, L^{*}\right)$-lsc (resp usc) and monotone increasing then

$$
f(X)=\sup _{Q \in L_{+}^{*} \mid Q(\mathbf{1})=1} R(Q(X), Q), \text { resp. } f(X)=\max _{Q \in L_{+}^{*} \mid Q(\mathbf{1})=1} R(Q(X), Q) .
$$

Proof. See [13] Proposition 5.4.

\section{References}

[1] B. Acciaio and I. Penner, Dynamic risk measures, Preprint (2009).

[2] C. D. Aliprantis and K. C. Border, Infinite Dimensional Analysis, 3rd edn. (Springer, Berlin, 2005).

[3] F. P. Y. S. Berrier, L. C. G. Rogers and M. R. Theranchi, A characterization of forward utility functions, Preprint (2007).

[4] F. Bellini and M. Frittelli, On the existence of minimax martingale measures, Mathematical Finance 12(1) (2008) 1-21.

[5] S. Biagini and M. Frittelli, A unified frameork for utility maximization problems: An Orlicz space approach, Ann. Appl. Prob. 18(3) (2008) 929-966.

[6] S. Biagini and M. Frittelli, On the extension of the Namioka-Klee theorem and on the Fatou property for risk measures, in Optimality and Risk: Modern Trends in Mathematical Finance, The Kabanov Festschrift, F. Delbaen, M. Rasonyi and CH. Stricker (eds.) (2009).

[7] S. Cerreia-Vioglio, F. Maccheroni, M. Marinacci and L. Montrucchio, Risk measures: Rationality and diversification, The Carlo Alberto Working Papers No. 100 (2009).

[8] M. H. A. Davis, Option pricing in incomplete markets, in Mathematics of Derivative Securities (Newton Institute, Cambridge University Press, 1997), pp. 216-226.

[9] F. Delbaen, P. Grandits, T. Rheinlander, D. Samperi, M. Schweizer and C. Stricker, Exponential hedging and entropic penalties, Mathematical Finance 12(2) (1997) 99123.

[10] N. El Karoui and R. Rouge, Pricing via utility maximization and entropy, Mathematical Finance 10(2) (2000) 259-276.

[11] N. El Karoui, S. Peng and M. C. Quenez, A dynamic maximum principle for the optimization of recursive utilities under constraints, Ann. Appl. Prob. 11(3) (2001) 664-693.

[12] H. Föllmer and A. Schied, Stochastic Finance, An introduction in discrete time, 2nd edn., de Gruyter Studies in Mathematics, Vol. 27 (2004).

[13] M. Frittelli and M. Maggis, Dual representation of quasiconvex conditional maps, Preprint (2009).

[14] M. Frittelli, Introduction to a theory of value coherent with the no arbitrage principle, Finance and Stochastics 4(3) (2000) 275-297.

[15] V. Henderson and D. Hobson, Horizon-unbiased utility functions, Stoch. Proc ES Appl. 117(11) (2007) 1621-1641.

[16] S. D. Hodges and A. Neuberger, Optimal replication of contingent claims under transaction costs, Re. Futures Markets 8 (1989) 222-239.

[17] H. Hudzik and Z. Zbaszyniak, Smoothness in Musielak-Orlicz spaces equipped with the Orlicz norm, Collect. Math. 48 (1997) 543-561.

[18] D. M. Kreps and E. L. Porteus, Dynamic choice theory and dynamic programming, Econometrica 47(1) (1979) 91-100. 
[19] H. Kunita, Stochastic Flows and Stochastic Differential Equations, Cambridge University Press (1964).

[20] A. Lazrak and M. C. Quenez, A generalized stochastic differential utility, Mathematics of Operations Research 28(1) (2003) 154-180.

[21] P. A. Meyer, Probability and Potentials (Blaisdell Publishing Col, London, 1966).

[22] M. Musiela and T. Zariphopoulou, Investment and valuation under backward and forward dynamic exponential utilities in a stochastic factor model, in Advances in Mathematical Finance, Applied and Numerical Harmonic Series (2006), pp. 303-334.

[23] M. Musiela and T. Zariphopoulou, Optimal asset allocation under forward exponential criteria, Markov Processes and Related Topics: A Festschrift for T.G. Kurtz, IMS Collections, Institute of Mathematical statistics 4 (2008) 285-300.

[24] J. Musielak, Orlicz Spaces and Modular Spaces, Lectures Notes in Mathematics, Vol. 1034 (Springer, 1983).

[25] A. Pratt, Risk aversion in the small and in the large, Econometrica 32(1/2) (1964) 122-136.

[26] R. T. Rockafellar, Integrals which are convex functionals, Pacific Journal of Mathematics 24(3) (1968) 525-539. 